\title{
Hallmarks of Cancer: Interpretation as Carcinogenic Hypercycle
}

\section{Malzev VN}

Department Practice of Urology, Bahnhofstr 1, 30159 Hanover, Germany

*Corresponding author: Malzev VN, Urologische Praxis, Bahnhofstr. 1, 30159 Hanover, Germany, Tel: +49 5113275 74; E-mail: wladimirmalzev@mail.ru

Received date: November 16, 2015; Accepted date: February 01, 2016; Published date: February 04, 2016

Copyright: () 2016 Malzev VN. This is an open-access article distributed under the terms of the Creative Commons Attribution License, which permits unrestricted use, distribution, and reproduction in any medium, provided the original author and source are credited.

\begin{abstract}
The mechanism that is the base of all hallmarks is the genetic instability as a result of continuously occurring mutations and egipenomic DNA modifications in a cancer cell. It cannot be explained by simple accumulation of genome mutations. Continuous mutation and epigenetic modification are only possible as a result of continuous impact of a mutagenic factor. Cyclic DNA replication reaction and/or RNA of mobile genetic elements are this mutagenic agent. These elements are generated as a result of inner-cell chaos of molecular biological processes that is caused by the impact of a cancerogenic factor. After their generation, they can create a hypercyclic link to the cell DNA replication cycle and hence cause mutations and epigenetic modifications in this cell. A new type of selforganisation of inner-cell processes and structures named primary cancerogenic hypercycle is generated. These changes progress, but remain hidden until they affect certain weak points of the cell genome. As a result, additional cyclic processes are generated that support the primary cancerogenic hypercycle and entail the generation of a second-order hypercycle. Hypercycles of the second order and over are hallmarks of cancer, and they ensure a competitive advantage with regard to the cyclic DNA replication reaction of environmental healthy cells. Since this moment, a pre-cancer cell becomes a cancerous one. The primary cancerogenic hypercycle remains a homogenous structure during the whole cancerogenesis. This hypothesis reveals new principles of cancer treatment that are described in the article.
\end{abstract}

Keywords: Carcinogenic hypercycle; Cell DNA replication limit cycle; Limit cycle bifurcation; Carcinogenesis

\section{Summary}

The article deals with the hypothesis of cancerogenesis that describes cancerogenesis as a cancerogenic hypercyle, a dissipative system that was generated by self-organisation of inner-cell processes after the chaos caused by cancerogenes. Hallmarks of cancer are interpreted as components of this cancerogenic hypercycle. The hypothesis of cancerogenic hypercycle is of integral nature.

\section{Introduction}

In 2000, a work [1] was published where the authors tried to describe the basic features characteristic for cancer. They have determined six of such basic principles. 10 years later, the same authors had to extend this list to ten [2] (Figure 1). But even this list is not complete. In the course of cancer research, new features unknown before are added. For example, a feature like the generation of long non-coding RNA [3] was added to the list. The gained ability of cancer cells to be insensitive to chemotherapy can be added to it as well [4].

We have also added to this list and centralised, besides the genetic instability 11, also p. 12. As the RNA world plays an important role in ensuring the genetic instability. Genetic instability is the main and primary feature of cancerous transformation, a characteristic innate to all malignant tumours; all other features are products of it. This is why the key for understanding and solving the cancer problem is to understand the mechanism of genetic instability of a cancerous cell.

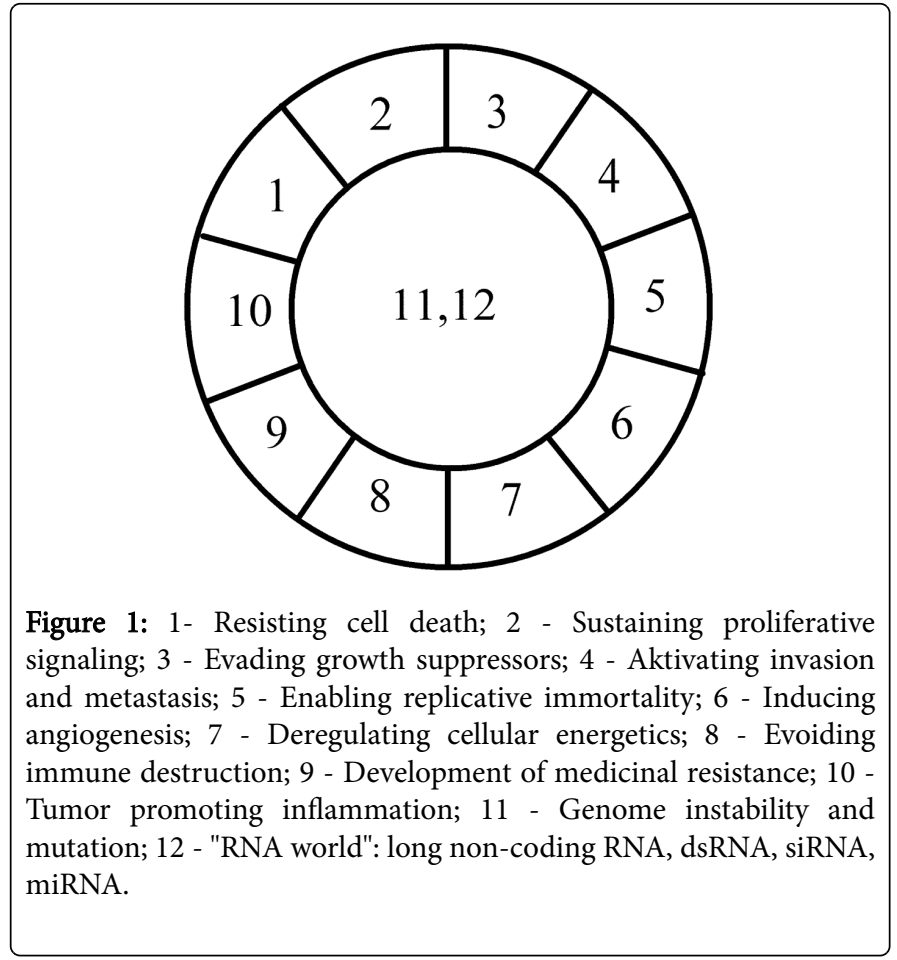

Genetic instability is innate not only to cancerous cells, but also to normal ones. In normal cells of eukaryotes, it exists as a somatic hypermutagenesis. Under the impact of mutagenic factors (AID, UDG and recombination complex components), mutations necessary for a normal function of the adaptive immunity take place in B cells DNA. 
The adaption is ensured by genetic DNA diversity of these cells as a result of mutations [5].

Bacteria also have such mechanism: If environment conditions modify, hypermutagenesis emerges, entailing DNA mutations and bacteria adaption that ensures their surviving under new conditions [6].

In both cases, somatic hypermutagenesis is not a continuously active factor: It is launched and controlled according to the neediness. In case of adaptive immunity according to need of organism immunity; in case of bacteria according to need to adapt to the environment. Besides, the active and targeted nature of the hypermutagenesis must be mentioned: It only takes place in case of impact of mutagenic factors. We do not deal with any spontaneous DNA errors accumulation.

Contrary to somatic hypermutagenesis of normal cells of eukaryotes and bacteria, in a cancerous cell, the hypermutagenesis is of continuous nature. This can only be explained by the fact that there is a hypothetic mechanism of mutagenic factor(s) self-reproduction. The simplest form of self-reproduction is a closed cyclic replicative process. Such process is presented (Figure 2).

The further discussion must be led by using the primary conceptual apparatus of synergetics and not only of biological disciplines as it better suits to describe the chaos processes taking place in a dividing cell, self-organisation, and the course of a dynamic processes.

\section{Limit Cycle of Cell DNA Replication: Possible Development Variants}

The division of any cell including a cancerous one is a cell cycle.

Any cyclic process including the cell cycle can be abstractly imagined as a limit cycle, i.e., closed curve (Figure 2) [7]. This curve is nothing different than the graphical image of cell DNA replication, presented as alternating and changing in time. Considering the fact that life is a molecular replicator continuous in time [8], such abstraction is quite correct.

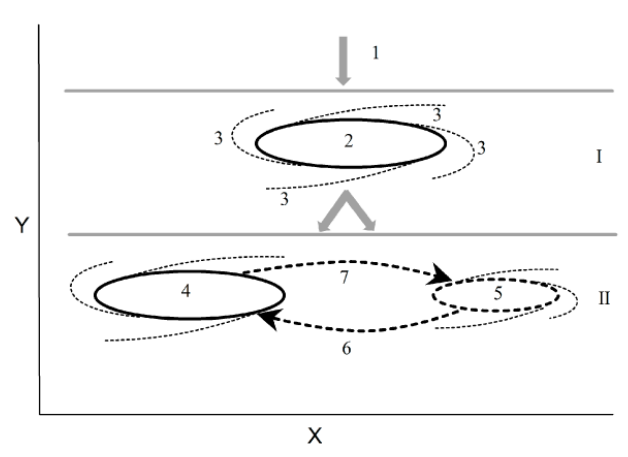

Figure 2: During the impact of the leading factor 1 on the limit cycle of the cell DNA replication 2, bifurcation of this limit cycle to two (or more) limit cycles takes place, one of which - the primary limit cycle 4 - is a cell DNA replication cycle, and the other one - the secondary cycle 5 - is an independent cycle of individual molecules of DNA or RNA replication.

1 - Leading factor; 2 - Cell cycle presented as a limit cycle of the cell DNA replication; 3 - Alternating molecule ensembles, components of the limit cycle; 4 - Primary limit cycle; 5 - Secondary limit cycle; 6 Mutagenic factor; 7 - Factor that provides resources for the secondary limit cycles.

In the reality, DNA cell replication is not realised on its own, but takes places with participation of sub-systems that are components of the replication cycle. This closed curve is created from a number of components, many alternating (Figure 2) [7]. In a real cell cyle, these alternating components include molecule ensembles, e.g., "protecting" proteins p53, cyclins, kinases, growth factors and cell organelles that take part in cell DNA replication in any way. The limit cycle is an attractor for all of its components, i.e., a trajectory to which all these components are attracted, to which their own trajectories are attracted, and is of dynamic nature. The latter means that the attractor function is not to be understood as something firmly fixed and determined. Trajectories of its components can modify under the impact of the leading factor, i.e. under exterior impact that includes e.g. temperature, radiation, impact of chemical factors or the environment $\mathrm{pH}$ (Figure 2). Furthermore, like for any dissipative system, fluctuation and sensitivity to initial conditions are characteristic for DNA replications; this determines the stochastics of this system [7]. As a result of the impact of these factors, the limit cycle can lose its stability. The trajectories of its alternating components modify, the limit cycle itself becomes "loose". This is expressed by the weakened ability of the attractor to attract the trajectories of its components. In molecular biology terms, this means that the cell organelles structure and functions are perturbed, such as mitochondria or the nucleus pore complex. In the latter ones, perturbation of major molecules transportation takes place due to affection of transport proteins (transporter proteins of the RNA, Nup214 and Nup88.) [9]. As a result of compartments affection, discoordination and perturbation of biochemical reactions and all types of exchange takes place, an oxidative choc emerges [10].

After that, bifurcation takes place (Figure 2), and the limit cycle splits to two or more limit cycles (Figure 2).

Such dynamic structure appears in one of the daughter cells as a result of stem cell mitosis after the impact of a cancerogenic factor on the mitosis process.

The destiny of such parallel limit cycle can have different dynamic forms. In molecular biology terms, this means that it can become a mobile genetic element and spread as a virus or persist as a virus in ribosomes (21), it can degrade and stop its existence, it can get in interaction with another secondary limit cycle in the form of interaction of two or more viruses.

We would like to mention that the dominating theory on virus generation confirming this assumption is the theory on endogenic virus generation, and, as a variant of this theory, the proretroviral theory explaining the generation of RNA viruses from mobile elements [11]. The fact that cell oncogenes are closely connected to viral oncogenes and are modified variants of normal genes also speaks in favour of this theory. Moreover, DNA proviruses and RNA proviruses that can serve as base for such parallel mutagenic limit cycle are integrated in the cell genome [12].

However, it must be mentioned that the secondary limit cycle is not a virus replication in the full sense of this word. It is missing the synthesis of components like protein and lipid cover. In this sense, it is more similar to viroid replication - a structure without the components mentioned above, or to ribosome replication. The secondary limit cycle can have different forms also if we cannot tell the exact virus-like form 
Page 3 of 7

but would like to express the phenomenon essence of a cyclic replication of this virus-like form in the virology language; we call it quasi-virus persistance, meaning one of the forms mentioned above.

We would like to mention that there is another generation mechanism of a secondary limit cycle, besides the DNA cell replication limit cycle bifurcation. This is self-organisation of "molecular garbage" of the cell cytoplasm in the form of any elements capable of replication, such as dsRNA or DI particles.

Finally, such limit cycle - and this is the essence of our hypothesis can create a hypercyclic link with the generation of a DNA cell replication hypercycle of primary order or a primary cancerous hypercycle. Any of these variants is of coincidental stochastic nature. The way of the further self-organisation of inner-cell processes depends on the ability of the DNA cell replication limit cycle to be an attractor for all components of its trajectories and on the ability of these components to be a competitor to it. Cyclic reactions less stable than a DNA replication under usual conditions, such as RNA replication cycle, can also be a competitor for this cycle. Under certain conditions, this cycle can displace and replace the DNA cell replication cycle. The conditions modifying the means of interaction of a DNA cell replication and a RNA replication were described in our precedent works $[13,14]$.

So, what is a hypercycle and of what nature is it?

A hypercycle is:

1. A means of merging of self-reproducing macromolecules in closed autocatalytic chemical cycles.

2. Several cyclic reactions organised in a way in which by-products of one reaction are catalysts for the other and the last reaction produces a catalyst for the first one.

3. A means of merging of self-reproducing units in a new, stable system capable of evolution [15].

4. A system that connects autocatalytic self-reproducing units with each other by a cyclic link.

In conditions of lack of energy resources “...The hypercycle competes with any self-reproducing unit that is not a member of it. It cannot coexist with other hypercycles either if not merged with them in an autocatalytic cycle of a next, higher order. Consisting of independent self-reproducing units it has integrating features. A hypercycle merges these units in a system capable of coordinated evolution, where benefits of one individual can be made use of by all its members." (Eigen).

A hypercycle is a means of self-organisation of material from chaos. It is precisely this why the hypercycle hypothesis has shown to be attractive to explain life generation [15]. With regard to applying this hypothesis to cancerogenesis, in case of cancer transformation we face inner-cell chaos generated under the impact of cancerogenic factors. The fact that cancer is a chaotic process is already confirmed [16]. This inner-cell chaos spreads by involving additional functional cyclic structures, firstly on the tissue level, then also on the level of the whole organism. In this sense, cancer is a step backwards in the evolution ladder, a return to the state of generation and development of life that has existed millions of years before the moment when resulting from competition and natural selection, the hypercycle has not formed in a relatively stable limit cycle of DNA replication.
We have discussed in a more detailed way in our previous works the questions of generation of parasite replicative reactions and their competition with a cell DNA replication cycle, replacing and displacing of this cycle by them and connected modifications in cell DNA replication (references) [13,14,17,18].

The more cyclic units are included in the hypercycle, the higher the hypercycle order, the more resources it can attract to ensure its existence, the stronger its competitiveness. Hallmarks of cancer are cyclic units that increase the hypercycle order. These additional structures act like the primary hypercycle: Activating the expression of a certain gene or epigenomic code by the corresponding enzyme entails enhancement of this gene or code - according to the principle of positive feedback - if you understand as this the behaviour of the whole hypercycle system and not of its components. Such effect of selfsimilarity is a universal law of nature characteristic for fractal structures that are characteristic for chaotic processes during their selforganisation. Hypercyclic features mentioned above are characteristic for these cyclic processes, just like for the primary hypercycle: 1 . Macromolecule self-reproduction (activation of a gene or epigenomic code entails production of macromolecules, and, in case of further replication of the whole hypercycle, their self-reproduction). 2 . Products of one reaction are catalysts for the other one (these macromolecules entail stimulation of cell DNA replication, and the cell DNA replication, in its turn, supports the production of these macromolecules). 3. These cyclic processes are merged with the primary cancerogenic hypercycle in a unique, stable system capable of evolution. 4. These cyclic structures are connected by a cyclic link (Figure 3).

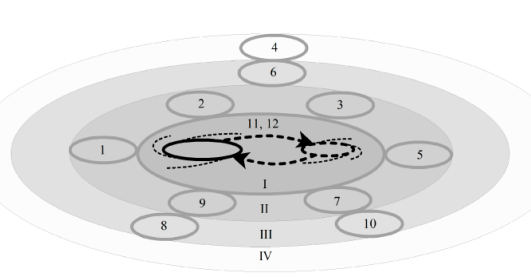

Figure 3: I - Primary cancerogenic hypercycle. 11 - Genome instability and mutation 12 - "RNA world": long non-coding RNA, dsRNA, siRNA, miRNA; II - Intracellular cyclic reactions. 1 Resisting cell death, 2 - Sustaining proliferative signaling, 3 Evading growth suppressors, 5 - Enabling replicative immortality, 7 - Deregulating cellular energetics, 9 - Development of medicinal resistance; III - Tissue cyclic reactions, 6 - Inducing angiogenesis, 8 Evoiding immune destruction, 10 - Tumor promoting inflammation; IV-Cyclic reactions at the level of an organism, 4 Activating invasion and metastasis.

Figure 3 shows a primary-order hypercycle formed from two cyclic processes mentioned above with participation of the "RNA world" [13] marked by No. 12. This hypercycle entails genetic instability of the cell DNA marked by No. 11. Resulting from this instability, cyclic processes are generated that enhance the primary-order hypercycle and form higher-order hypercycles that run on the level of inner-cell processes and are marked by No. II. Cyclic processes that develop on the tissue level and that enhance I as well as II are marked by No. III. Finally, cyclic processes that run on the organism level and have a system impact on it are marked by No. IV. All of them are a feed-back circuitry and correspond to hallmarks of cancer (Figure 1). 
Page 4 of 7

In case of cancerogenesis, the parallel secondary cycle produces a certain mutagenic factor that has an impact on the primary limit cycle. Or, spoken differently, the secondary mutagenic cyclic process 6 has a mutagenic impact stable in time on the primary cyclic process 5 (Figure 2). The primary limit cycle produces a factor/factors that, in its/their turn, support(s) the secondary limit cycle. This is what the cancerogenesis scheme based on the simplest primary abstract hypercycle looks like.

The mutagenic factor itself can be of different nature, and this nature depends on the secondary limit cycle structure: The mutagenic factor can be the enzyme able to remodel the chromatin, a long noncoding RNA causing epigenomic modifications as well as siRNA also able to modify the cell epigenomic code. For example, in case of creation of a secondary limit cycle based on DNA, the replication of the latter one can take place with creation of an enzyme like chromatin remodelling enzyme of a normal cell $\mathrm{CHD} 1$ and $\mathrm{CHD} 2$ chromatin remodelling enzymes [19]. In case of creation of a hypercycle based on RNA interference, siRNA will be such mutagenic factor [14].

However, a mutagenic factor does not only mean the impact of some substance on DNA, but also indirect impact of a secondary limit cycle on the primary limit cycle. Consuming the energetic resources of this cell as well as resources of the cell connected to protein translation in ribosomes - the ribosomes are busy to produce enzymes necessary for the secondary limit cycle - this secondary limit cycle can at a certain stage cause perturbation of the DNA repairing mechanism due to suppression of the activity of its repairing enzymes. The suppression of the repairing enzymes activity as well as their increased activity in cancer at the example of DNA polymerase is described in literature [20].

The situation is even aggravated if the bioenergetics of the cancerous cell is perturbed, when the oxidising phosphorylation is changed by anaerobic glycolysis, which entails an even greater deficit of energetic resources. Besides, the suppression of the repairing enzymes expression can take place due to epigenomic impact of a secondary limit cycle on the cell DNA.

This idea can be confirmed by examples of interaction between cell replication and virus replication as two competing replicative units not connected by a hypercyclic link. Doing so, viruses have the following impact on the cell:

Viruses block the transcription processes; Viruses cause chronic inflammation in the cell [21]; Viruses suppress the apoptosis [22,23]; Viruses activate mitosis, and the factor causing such activation is not DNA cell mutation but the virus developing process in the cell itself [24].

Hence, even simple competition of a replicative cyclic unit in the form of a virus in the cell entails emergence of some features characteristic for cancer in it. Especially tumour promoting inflammation marked by No. 10 on Figure 1, resisting cell death marked by No. 1 on Figure 1, mitosis activation under No. 2 and 3 on Figure 1.

It is remarkable that it is especially in case of apoptosis suppression that viruses gain the ability to persist, and hence, for cell malignation.

\section{Means of viruses interaction as interaction model of replicative cyclic units in a hypercycle}

Viruses in a cell interact by complementation and recombination.
Complementation is a virus interaction where one of them or both provide missing proteins for reproduction and development to the other one. This means essentially a hypercycle expressed in virology by the term "complementation" if we assume that the virus produces an enzyme also for its own replication. Hence, we have the evidence for the fact that two replicative units are able to get in a hyper cyclic link between each other. What is fair for virus-virus interaction is also fair for virus-DNA cell replication cycle interaction.

\section{Hypercycle Structure and Dynamics}

If a tumour consists of heterogeneous mutating cell clones, the primary hypercycle regarded as interaction of the primary and secondary limit cycle is probably a homogeneous dynamic structure characteristic for all these cell clones. The fact that the epigenetic image of human bowel cancer mutations corresponds to the one of mice speaks in favour of this assumption [25]. This indicates identic cancerogenesis dynamics of so different organism species. Such identic dynamics can only be ensured by an identic hypercycle dynamic system.

Besides, the cancerogenesis is a multi-level process that is expressed in gradual involving of new cyclic units on each level as a result of cell division. Doing so, the interaction of all its cyclic structures entails stabilisation and reinforcement of the primary hypercycle determined as interaction of the primary limit DNA cell replication cycle and the secondary limit cycle. This primary hypercycle remains unmodified in all cell clones thanks to support from additional cyclic units and natural selection. This is why, according to the nature of this primary hypercycle, all mutant tumour clones are homogeneous.

The duration of the secondary limit cycle replication depends on its content: The larger the molecules subject to replication, the longer lasts the cycle. This conclusion results from the duration of replication of different virus species in a cell. DNA viruses require more time for their replication than RNA viruses. This is why the replication cycle of a secondary DNA containing limit cycle can be compared to a DNA virus replication cycle. The replication speed of the latter one is approx. 22 hours and can be compared to the average speed of the replication of the cell itself, with regard to the duration. Taken a RNA virus replication, it is shorter than a DNA virus replication and is already 6-12 hours, what is 2-3 times faster than a cell replication cycle [26].

Speaking about short dsRNAs replication, their replication cycle is even shorter. If, in case of the DNA containing limit cycle, we can speak about a cell replication synchronous with the DNA, then in case of RNA, there is no such synchronous replication. A surplus product is created that - if it does not start further replication - must either be utilised by the cell or be bounced out by it; or else the new RNA replicant must be substandard, i.e. not be able to continue to replicate, what can be the case in its too radical mutation. An example for the last variant is RNA mutating into DI particles unable to replicate, but able to compete with wholesome RNA due to their smaller size compared to theirs, by occupying their place in the transcription apparatus. Hence, they have a depressing impact on the secondary limit cycle RNA replication. This balancing mechanism can synchronise replication processes of the cell DNA and of the secondary limit cycle. In case of fast dsRNA replication, its surplus in the form of siRNA is just bounced out of the cell.

In both cases, hyperbolic growth does not take place, just like in a virus replication in a cell with saturation until a certain limit due to limited resources of the host cell. 
Page 5 of 7

A RNA surplus product can be a good diagnostic criterion for cancer. An example is siRNA and other RNAs in cancer [27].

Division speed of a cancer cell does not exceed the division speed of a normal cell. This is why the primary limit cycle period - the cell DNA replication - is in a certain way dynamically coordinated with the secondary mutagenic limit cycle replication period. Hence, we have to do with a variant of an oscillator, when the curve of one process meets the curve of the other one.

Another mechanism of speed balance of the secondary limit cycle replication and the cell DNA replication is unlimited replication of the latter one, i.e. uncontrolled cell division. The cell replication speed corresponds with the secondary limit cycle replication speed. This is the difference from multiplication of a virus in a cell or a phage in a bacterium. In the case when there is no such corresponding, the cell dies or becomes substandard for further replication. From the point of view of this hypothesis, observed pathologic mitoses in tumours can be interpreted as a result of the division of such substandard cells. As a result of such natural selection, only the cells the hypercycle dynamics of which is balanced by their replication speed survive and continue the tumour progression.

Cancer cell division takes place in geometric progression. This geometric division progression corresponds to the speed and replication of the secondary limit cycle as a component of a cancerogenic hypercycle, and hence, obstructs the cell saturation by this secondary limit cycle. It must be remembered that contributing as well as obstructing factors of development of one replicative hypercycle cyclic units in a cell are various. DI particles and different RNA types can be included: cyclic RNAs, siRNAs, miRNAs, long non-coding RNAs as well as pseudogene transcripts. Pseudogene transcripts, long non-coding RNAs, cyclic RNAs are more active as internal competing RNAs than mRNAs. mRNAs, long non-coding RNAs and pseudogene RNAs can be subject to destruction in interaction with microRNAs, and cyclic RNAs are more stable when connecting with microRNAs [28].

\section{Falsifiability of the Hypothesis of Cancerogenic Hypercycle}

Considering the non-lined nature and stochastics of processes taking place in the cell dissipative system, the hypothesis can be confirmed by computer modelling with following testing on a biological model. A biological model can be an animal as well as a cancer cells culture. A cancer cell culture is a simplified and hence more accessible for computer modelling biological model, compared to an animal biological model as, if used, the impact of cyclic process groups marked on Figure 3 by No. III (Tissue cyclic reaction) and IV (Cyclic reactions at the level of an organism) are excluded. Having excluded the impact of these cyclic processes, a primary cancerogenic hypercycle can be modelled more precisely than in the case of an animal.

\section{Treatment Principles}

A radical chemotherapeutic treatment requires measures targeted to suppress the ability of cancerous cells to mutate, i.e., against genetic instability. It is only in case of suppression of this ability and elimination of the genetic instability that we can count on radical healing. Considering that the source of hypermutagenesis of a cancerous cell is a primary cancerogenic hypercycle as well as the above-mentioned fact that this primary cancerogenic hypercycle is the same for all cancerous cells clones, we can assume that the destruction of this hypercycle will have a therapeutic impact on all mutant tumour clones, and this kind of treatment can show to be radical.

The cancerogenic hypercycle can be destroyed by stabilising the primary limit cycle of the cell DNA replication, and the competitiveness of this limit cycle must be increased, compared to other replicative cyclic reactions. This goal can be achieved on several ways:

Treatment by disconnecting the hypercyclic link can be performed depending on the cancerogenic hypercycle nature.

If the base for the cancerogenic hypercycle is replication of the secondary DNA containing limit cycle, and an enzyme-mutagen ensures mutagenic effect on cell DNA, a substance must be injected that depresses this enzyme. If the base for it is long non-coding RNA replication, a substance that blocks the RNA replicase or a substance similar to DI particles of viruses is required to block the transcription.

If the base for it is dsRNA replication, a substance must be injected that blocks the siRNA as an agent competing by RNA interference. Moreover, a substance can be injected that destroys dsRNA and hence interrupts the hypercyclic link. Such types of chemical agents that have an impact on siRNA and on dsRNA include cytotoxic ribonuclease. It has already successfully passed tests and has shown a therapeutic antitumour effect under the name onconase $[29,30]$. Another way to block the hypercyclic link in a cancerogenic hypercycle of this type is blocking the RNA-dependent RNA polymerase. The anti-tumour effect of inhibiting the RNA-dependent RNA polymerase is already proven. This effect is expressed in case of combining chemotherapy and such blockade, i.e., according to the way mentioned above [31]. Pyrogallol also has such inhibiting impact [32]. The anti-tumour effect of pyrogallol is already proven on the example of lung cancer treatment [33].

In the case when the cancerogenic hypercycle was generated based on dsRNA replication, there is an interesting possibility to control the treatment effectivity by studying the siRNA and miRNA profiles on their different stages.

Treatment by creating a competing limit cycle for the cancerogenic hypercycle

As a result of such competition, cell resources are consumed by this competing unit, and due to this, the cancerogenic hypercycle is depressed. An example of such treatment can be infection of a melanoma cancer cell by a genetically modified virus [34,35].

A method that combines the two first ones: Disconnecting the hypercyclic link of a cancerogenic hypercycle with injecting a genetically modified virus that, by complementation and recombination, could deviate the parallel secondary limit cycle trajectory and connect it to oneself, and hence disconnect the cancerogenic hypercycle. Theoretically, such method could be effective in case of cancerogenesis induced by endogenic retroviruses and other RNA-containing viruses as a high recombination level is characteristic for them [12].

A method targeted to destroy the synchronisation of the primary and the secondary limit cycle. It means to inject factors that decelerate the RNA replication by competing antagonism into the cell. Such factors include DI particles, pseudogene transcriptors, cyclic RNAs. 
Finally, by performing modern target therapy and blocking cyclic units of presented hallmarks of cancer, we decrease the hypercycle level and its ability to compete with the DNA replication limit cycle of healthy environmental cells. These methods include application of HGF/ c-met inhibitors, PARP inhibitors, inhibitors of VEGF signalling, selective anti-inflammatory drugs, telomerase inhibitors, immune activating anti-CTLA4 mAb, cyclin-dependent kinase inhibitors, EGFR inhibitors, aerobic glycolysis inhibitors, proapoptotic $\mathrm{BH} 3$ mimetics [2].

However, such ways of treatment do not eliminate the cancerogenic hypercycle, but only decrease the level of its competitiveness. Just like classic chemotherapy, they can prolong the patient's life affected by a propagated cancer form, but cannot radically cure him/her.

In the conclusion, very rare but anyway scientifically proven cases of spontaneous self-curation from cancer must be mentioned [36-38]. The only explanation of this phenomenon known to us is the successful organism immunity. The hypothesis proposed by us gives another possible explanation. The essence of it is the fact that all mutant tumour clones are homogenous, according to the nature of the primary cancerogenic hypercycle. And, if the patient's organism produces a sufficient amount of natural inhibitors for this cancerogenic hypercycle, such as pseudogene transcriptors, DI particles or cyclic RNAs, then the process of genetic instability and continuous mutagenesis in all cancer cells can be stopped and a spontaneous curation occurs.

\section{Cancer Detection and Treatment Effectivity Control}

Cancer detection and treatment effectivity control can be realised by cancerogenic hypercycle modelling based on its alternating components. They include the inner-cell enzymes activity ensuring DNA and RNA replication, the cyclic RNA, siRNA, miRNA level, long non-coding RNA, pseudo-genes transcripts, DI particles.

\section{Acknowledgement}

The authors would like to thank Aljona Malzev, for valuable support \& skill in translating the manuscript from Russian to English.

\section{References}

1. Hanahan D, Weinberg RA (2000) The hallmarks of cancer. Cell 100: 57-70.

2. Hanahan D, Weinberg RA (2011) Hallmarks of cancer: the next generation. Cell 144: 646-674.

3. Gutschner T, Diederichs S (2012) The hallmarks of cancer: a long noncoding RNA point of view. RNA Biol 9: 703-719.

4. Svirnovski AI (2011) Tumor cell resistance to therapeutic factors as medicobiological problem. Med. News

5. Teng G, Papavasiliou FN (2007) Immunoglobulin somatic hypermutation. Annu Rev Genet 41: 107-120.

6. Bridges BA (2001) Hypermutation in bacteria and other cellular systems. Philos Trans R Soc Lond B Biol Sci 356: 29-39.

7. Haken H (1985) Synergetics: Hierarchies of Instabilities in SelfOrganising Systems, Moscow.

8. Koonin EV (2012) The Logic of Chance. The Nature and Origin of Biological Evolution. Izdatel?stvo Centrpolygraph.

9. Köhler A, Hurt E (2010) Gene regulation by nucleoporins and links to cancer. Mol Cell 38: 6-15.
10. Scholz W, Schütze K, Kunz W, Schwarz M (1990) Phenobarbital enhances the formation of reactive oxygen in neoplastic rat liver nodules. Cancer Res 50: 7015-7022.

11. Izmaylova JS (1995) Development of Basic Theories of Generation and Evolution of Viruses. Chief Certificate Commission of Russian Federation 07.00 .10

12. Fields BN, HS Knipe DM, Murphy FA (1989) Fundamental Virology. Moscow

13. Malzev VN (2013) Cancer as a carcinogenic hyper-cycle and a RNA world progress. Med Hypotheses 81: 288-292.

14. Malzev VN (2014) New cancer systematization: hypothesis of the carcinigenic hypercycle in Book RNA Viruses: Emergence, Classification and Public Health Concerns. Nova Science Publishers, Inc.

15. Eigen M, Schuster P (1982) The hypercycle. A Priciple of Natural SelfOrganization. Springer publishing house.

16. Pham TD, Ichikawa K (2013) Spatial chaos and complexity in the intracellular space of cancer and normal cells. Theor Biol Med Model 10: 62.

17. Malzev VN (2013) Cancerogenic hypercycle: a new conception of cancer treatment. OA Medical Hypothesis 1:12.

18. Malzev VN (2013) Cancer as a carcinogenic hyper-cycle and a RNA world progress. Med Hypotheses 81: 288-292.

19. Siggens L, Cordeddu L, Rönnerblad M, Lennartsson A, Ekwall K, et al. (2015) Transcription-coupled recruitment of human CHD1 and CHD2 influences chromatin accessibility and histone $\mathrm{H} 3$ and $\mathrm{H} 3.3$ occupancy at active chromatin regions. Epigenetics Chromatin 8: 4.

20. Ren C, Cho SJ, Jung YS, Chen X (2014) DNA polymerase $\hat{I}$. is regulated by poly(rC)-binding protein 1 via mRNA stability. Biochem J 464: 377-386.

21. Zhukova O (2006) Molecular mechanisms of lymphocyte apoptosis injury in chronic viral infection. Bull. Sib. Med.

22. Viruses: Viruses: virus cytopathic effect and inhibition of apoptosis. Knowl. Base Mol. Biol. Gen. Hum. HUMBIO

23. Kusnecov TI, Urazov LN, Vidyaev IG (2006) Research on Virus-Induced Apoptosis of Tumour Cells in Vitro. S0069b. Oncol. J. 20:

24. Banerjee S, Narayanan K, Mizutani T, Makino S (2002) Murine coronavirus replication-induced p38 mitogen-activated protein kinase activation promotes interleukin- 6 production and virus replication in cultured cells. J Virol 76: 5937-5948.

25. Grimm C, Chavez L, Vilardell M, Farrall AL, Tierling S, et al. (2013) DNA-Methylome Analysis of Mouse Intestinal Adenoma Identifies a Tumour-Specific Signature That Is Partly Conserved in Human Colon Cancer. PLoS Genet 9: e1003250.

26. Zverev VV BMN (2010) Medical Microbiology, Virology and Immunology. GEOTAR-Media, Moscow.

27. Ravo M, Cordella A, Rinaldi A, Bruno G, Alexandrova E, et al. (2015) Small non-coding RNA deregulation in endometrial carcinogenesis. Oncotarget 6: 4677-4691.

28. Vaganova A Traps for miRNA - competing endogenous RNAs. Life Sci. Prog.

29. Saxena A, Saxena SK, Shogen K (2009) Effect of Onconase on doublestranded RNA in vitro. Anticancer Res 29: 1067-1071.

30. Zhao H, Ardelt B, Ardelt W, Shogen K, Darzynkiewicz Z (2008) The cytotoxic ribonuclease onconase targets RNA interference (siRNA). Cell Cycle 7: 3258-3261.

31. Fukushima H, Abe T, Sakamoto K, Tsujimoto H, Mizuarai S, et al. (2014) 3' ethynylcytidine, an RNA polymerase inhibitor, combined with cisplatin exhibits a potent synergistic growth-inhibitory effect via Vaults dysfunction. BMC Cancer 14: 562.

32. Koslov MV, Polyakov KM, Ivanov AV, Filippova SE, Kuzyakin AO, et al. (2006) RNA-dependent RNA polymerase of the hepatitis C virus: research of inhibiting mechanism of pyrogallol derivatives. Biochemistry (Mosc) 71: 1253-1259. 
Citation: Malzev VN (2016) Hallmarks of Cancer: Interpretation as Carcinogenic Hypercycle. J Carcinog Mutagene 7: 252. doi: $10.4172 / 2517-2518.1000252$

Page 7 of 7

33. Yang CJ, Wang CS, Hung JY, Huang HW, Chia YC, et al. (2009) Pyrogallo induces G2-M arrest in human lung cancer cells and inhibits tumor growth in an animal model. Lung Cancer 66: 162-168.

34. Woller N, Gürlevik E, Ureche CI, Schumacher A, Kühnel F (2014) Oncolytic viruses as anticancer vaccines. Front Oncol 4: 188.

35. Gujar SA, Pan DA, Marcato P, Garant KA, Lee PW (2011) Oncolytic virus-initiated protective immunity against prostate cancer. Mol Ther 19: 797-804.

36. Kim SB, Kang W, Shin SH, Lee HS, Lee SH, et al. (2015) Spontaneous neoplastic remission of hepatocellular carcinoma. Korean J Gastroenterol 65: 312-315.
37. Matsuoka S, Tamura A, Moriyama M, Fujikawa H, Mimatsu K, et al. (2015) Pathological evidence of the cause of spontaneous regression in a case of resected hepatocellular carcinoma. Intern Med 54: 25-30.

38. Tamás L, Sári E, Répássy G, Szabó P, Bagdi E, et al. (2011) Spontaneous remission in localized diffuse large B-cell lymphoma. Pathol Oncol Res 17: 779-784. 